\title{
Is the Deficit in Pain Inhibition in Fibromyalgia Influenced by Sleep Impairments?
}

\author{
Emilie Paul-Savoie ${ }^{1}$, Serge Marchand ${ }^{1}$, Mélanie Morin ${ }^{1}$, Patricia Bourgault ${ }^{2}$, Nathalie Brissette ${ }^{1}$, \\ Vongmaly Rattanavong ${ }^{1}$, Christian Cloutier ${ }^{1}$, Alain Bissonnette ${ }^{3}$ and Stéphane Potvin ${ }^{*}, 4$ \\ ${ }^{I}$ Department of Surgery, Faculty of Medicine and Health Sciences, Université de Sherbrooke; Sherbrooke, Québec, \\ Canada \\ ${ }^{2}$ Centre de recherche Fernand-Seguin, Department of Psychiatry, Faculty of Medicine, Université de Montréal; \\ Montréal, Québec, Canada \\ ${ }^{3}$ School of Nursing, Faculty of Medicine and Health Sciences, Université de Sherbrooke; Sherbrooke, Québec, Canada \\ ${ }^{4}$ Clinique du Campanile; Québec, Québec, Canada
}

\begin{abstract}
It has been proposed that a deficit in inhibitory conditioned pain modulation (ICPM) underlies the pathophysiology of fibromyalgia (FM), but there is high variability in ICPM efficacy in this syndrome that remains poorly understood. Based on emerging data showing that age, anxiety, depression and sleep can modulate ICPM efficacy, the main objective of this study was to determine the clinical correlates of experimentally-induced pain perception in FM. Fifty FM patients and 39 healthy controls (HC) were tested. Anxiety, depression, sleep and FM symptoms were measured with questionnaires or interview-type scales. Experimental pain testing consisted of two tonic heat pain stimulations separated by a 2-minute cold pressor test (CPT). Thermal pain thresholds and tolerance were higher in HC compared to FM patients. Pain ratings during the CPT were lower in HC relative to FM patients. ICPM efficacy was stronger in HC compared to FM patients. Finally, sleep quality was the only factor significantly related to ICPM efficacy. To our knowledge, this is the first study to report this association in FM. Future studies will need to replicate this finding, to determine whether impaired sleep is primary or secondary to deficient pain inhibition, and to characterize the neurobiological mechanisms underlying this association.
\end{abstract}

Keywords: Fibromyalgia, sleep, pain inhibition.

\section{INTRODUCTION}

Fibromyalgia (FM) syndrome is a chronic pain disorder of unknown etiology affecting an estimated $2 \%$ of the U.S. population [1]. This syndrome is characterized by widespread pain for at least 3 months combined with tenderness at palpation to $\geq 11$ of 18 specific tender points [2], although new non-tender point diagnostic criteria have been created [3]. Symptoms such as sleep disturbances, fatigue, anxiety and depressive symptoms are frequently associated with FM [4]. The complex clinical profile observed in FM highlights the heterogeneity of this disorder [5-8].

It has been proposed that FM results from altered endogenous pain modulation systems [9]. Pain is a dynamic phenomenon resulting from the activity of both endogenous pain excitatory and inhibitory systems, including inhibitory conditioned pain modulation (ICPM) [10]. The ICPM theory postulates that a nociceptive stimulation will cancel out another nociceptive stimulation if it occurs on a body surface

\footnotetext{
*Address correspondence to this author at the Centre de recherche FernandSeguin; 7331 Hochelaga; Montréal, Québec, H1N 3V2, Canada; Tel: 1-514251-4015, Ext. 2851; Fax: 1-514-251-2617;

E-mail: stephane.potvin@umontreal.ca
}

distanced from the pain surface [11,12]. This system involves serotoninergic, noradrenergic and opioidergic inhibitory pathways [11-14] and causes a diffuse reduction of pain throughout the body. On empirical grounds, some experimental studies have shown that ICPM is deficient in FM $[9,15,16]$.

Despite mounting evidence linking deficient ICPM to FM, there is high variability of ICPM efficacy in FM $[17,18]$. That is, FM may not only be associated with clinical but also with mechanistic heterogeneity. Unfortunately, the relationships between clinical variables and psychophysical measures in FM remain poorly understood, and the studies on the topic have focused mostly on (mechanical/thermal) pain thresholds, which are markedly reduced in FM $[17,19]$. Such studies have shown that tender point counts and thermal pain thresholds are significantly influenced by sleep quality, anxiety, depression and self-reported pain [20-22]. By comparison, few studies have explored the relationships between ICPM and clinical variables in humans, including only one study performed in FM [17].

Emerging pre-clinical and clinical data suggest that ICPM efficacy is influenced by socio-demographic and psychological factors such as anxiety, depression, age, and sleep quality [17,23-25]. For instance, our laboratory 
recently found that FM patients with depressive symptoms have more pronounced ICPM deficits, compared with nondepressive FM patients [17]. Similarly, anxiety may also be related to ICPM efficacy, given that the midbrain periacqueducal gray - a major ICPM brain structure critically regulates stress and anxiety in rodents [23]. Age has also been shown to modulate ICPM in healthy adults $[24,26]$. Finally, sleep quality also influences ICPM. Indeed, Smith et al. [25] altered healthy subjects' sleep for 7 nights and showed that sleep continuity disturbances impair endogenous pain inhibition.

The main objective of this study was to establish if experimentally-induced pain perception, especially ICPM efficacy, varies in function of depression, anxiety, age and sleep quality in FM. We also sought to confirm that FM is associated with reduced pain thresholds/tolerance and deficient ICPM.

\section{MATERIALS AND METHODOLOGY}

\section{Participants}

Fifty female patients suffering from FM and 39 female healthy controls (HC) participated in this study. All subjects were Caucasians. Patients were diagnosed with FM using American College of Rheumatology criteria (1990) [2] by neurosurgeons, rheumatologists or physicians on the ward, specialized in the treatment of chronic pain. None of the FM patients were referred by psychiatrists. Exclusion criteria were the following: (i) participants who were pregnant or breastfeeding; (ii) any clinically meaningful unstable, renal, hepatic, cardiovascular, respiratory, cerebrovascular disease or other serious, progressive physical illness; and (iii) participants who had diabetes. Among FM patients, 8 were diagnosed with comorbid major depressive disorder (MDD), based on a psychiatric interview performed by trained physicians (AB, CC) using DSM-IV criteria. FM patients and $\mathrm{HC}$ did not differ in terms of age $[\mathrm{FM}=49.8 \pm 10.5$; $\mathrm{HC}=50.0$ years $\pm 7.4 ; \mathrm{F}=0.01 ; \mathrm{p}=0.938]$ and the presence or absence (menopause or hysterectomy) of a regular menstrual cycle $[\mathrm{FM}=11$ presence, 39 absence; $\mathrm{HC}=9$ presence, 28 absence; $\left.\chi^{2}=0.07 ; p=0.799\right]$. FM patients were treated with one or more of the following medications [antidepressants $=$ 33; anti-inflammatory $\operatorname{drugs}=31$; anticonvulsivants $=22$; opioids=28; anxiolytics $=18$ ], and none were drug-free. None of the $\mathrm{HC}$ received psychiatric or pain medications. The Human Ethics Committees of the Centre hospitalier universitaire de Sherbrooke approved the research protocol, and all participants gave their written, informed consent.

\section{Psychological Factors}

\section{Severity of FM Symptoms}

Severity of FM was measured with the French version of Fibromyalgia Impact Questionnaire (FIQ) [4]. This selfadministrated questionnaire measures the components of health most affected by FM over the past week and contained 10 items (physical functioning, work status, depression, anxiety, sleep, pain, stiffness, fatigue, wellbeing) [4]. The French version is widely used by researchers and clinicians and has acceptable internal consistency, testretest reliability and construct validity [27].

\section{Depressive Symptoms}

Depressive symptoms in FM patients were measured using the French version of the Hamilton Depression Rating Scale (HAM-D). The HAM-D is a 17-questions multiple choice interview-type questionnaire [28]. This questionnaire is one of the most commonly used scales for rating depression in medical research. The HAM-D rates the severity of symptoms observed in depression such as change in mood, insomnia, agitation and weight loss. The HAM-D was administered by a nurse trained by SP, who has vast experience in administration of psychiatric interviews for research purposes [29-34].

\section{Anxiety}

Anxiety in FM patients was measured using the French version of the Hamilton Anxiety Rating Scale (HAM-A). The HAM-A is a 14-item questionnaire measuring the severity of anxiety symptoms [35]. This interview-type questionnaire provides measures of overall anxiety, psychological distress (psychic anxiety), and physical complaints related to anxiety (somatic anxiety). The HAM-A was administered by a nurse trained by SP, who has vast experience in the administration of psychiatric interviews for research purposes [29-34].

\section{Sleep Quality}

Sleep Quality in all participants were measured with the French version of Pittsburgh Sleep Quality Index (PSQI). The PSQI is a widely used, well-validated 19-item measure of sleep quality over the past month [36]. The PSQI is composed of seven clinically derived components of sleep difficulty, including subjective sleep quality, sleep latency, sleep duration, sleep efficiency, sleep disturbances, sedative medication use and daytime functioning, all of which are summed to a single global score.

\section{Pressure Pain Thresholds (PPT)}

Pressure Pain thresholds at tender points were assessed by a trained investigator, using a digital force gauge with a $1-\mathrm{cm}^{2}$ rubber tip (J-Tech Medical, Commander Algometer). Pressure was applied at a rate of $1 \mathrm{~kg} / \mathrm{s}$ on four specified tender points (left and right trapezoid, left and right knee). Subjects were instructed to verbally report when their sensations changed from pressure to pain. A mean tender point threshold $(\mathrm{kg})$ was calculated from four points. Healthy women usually start to perceive pain when $4 \mathrm{~kg}$ of pressure (or more) is given [2].

\section{Thermal Pain Measures}

\section{Thermal Pain Thresholds/Tolerance}

Thermal pain thresholds (TPT) and thermal tolerance (TOL) were measured by applying a thermode on the left forearm of participants. The Peltier thermode used (TSA II, Medoc, Advanced medical systems, Minneapolis, MN 55435) was a heating plate connected to a computer allowing a precise setting of temperature. Experimental temperature was initially set at $32^{\circ} \mathrm{C}$ and was gradually increased by a rate of $0.3^{\circ} \mathrm{C}$ per second. Subjects were instructed to verbally report when their sensations changed from heat to pain (TPT) and when their pain became intolerable (TOL). For each subject, the procedure was repeated 3 times to ensure the stability of TPT and TOL measurement. 


\section{Inhibitory Conditioned Pain Modulation (ICPM)}

Afterwards, a continuous heat pulse was administered with a thermode for 2 minutes on the left forearm of participants. Experimental temperature reached a predetermined fixed value and remained constant during the 2minute testing period (Time 0 to Time 120). It was set at a value corresponding to a temperature individually predetermined to induce a 50/100 pain rating during the pre-test [37]. During thermal stimulation (test-stimulus), pain intensity was measured using a computerized visual analog scale (COVAS), which ranged from 0 (no pain) to 100 (most intense pain tolerable). To capture the effects of inhibitory ICPM, we administered the test-stimulus twice and conducted a cold-pressor test (CPT) between administrations [38]. The CPT consisted in the immersion of the opposite (right) arm (up to the shoulder) for 2 minutes in a bath of cold water. Temperature of the water was set at $12{ }^{\circ} \mathrm{C}$ to ensure that the CPT (conditioning stimulus) was sufficiently painful to elicit ICPM while tolerable for 2 minutes. During the conditioning stimulus, subjects rated verbally their pain intensity every 15 seconds using a numerical pain rating scale, also ranging from 0 to 100 . The CPT recruits inhibitory ICPM because it is a strong nociceptive stimulation, which takes place during a lengthy time span [39] and is applied over a large body surface area [40]. By comparing the pain evaluation during the test-stimulus (2minute thermode) acquired before and after the CPT, we were able to measure the inhibitory ICPM response.

\section{Statistical Analyses}

Based on previous works from our group [17, 19], we expected FM patients to have a deficit in pain inhibition of a large magnitude. Using an effect size of $\mathrm{f}=0.4$, and a $\alpha$-value of 0.05 , the inclusion of $50 \mathrm{FM}$ patients and 39 controls conferred substantial statistical power $(\beta=96 \%)$ to our study. To investigate the potential differences in pain perception/modulation between FM patients and $\mathrm{HC}$, we performed analyses of variance (ANOVA), using group as the independent variable, and the following psychophysical measures as dependent variables: TPT, TOL, PPT ICPM efficacy (percentage of change between post- and pre-ICPM mean COVAS scores Time 0-120) and pain ratings during the CPT. Potential between-groups differences in age and menstrual cycle were examined using an ANOVA and the chi-square test, respectively. Potential relationships between clinical variables (age, FIQ total score, FIQ pain item, HAMD total score, HAM-A total score and PSQI total score) and psychophysical measures (PPT, TPT, TOL, pain ratings during the CPT, PPT, ICPM efficacy) were examined using Pearson's correlation analyses. Multiple linear regression analyses were performed when appropriate. That is, multiple regression analyses were performed when multiple clinical variables were related $(\mathrm{p}<0.1)$ to pain measures. Such analyses were also performed in order to rule out the influence of potential confounders (e.g. medications). For all analyses, the level of significance was set at $\mathrm{p}<0.05$.

\section{RESULTS}

\section{Participants Characteristics}

All screened participants, $50 \mathrm{FM}$ patients and $39 \mathrm{HC}$, completed the study protocol. HC did not complete the FIQ,
HAM-D, HAM-A and PSQI. Average scores for each psychological factor are shown in Table 1. FM patients receiving antidepressants had an elevated FIQ total score $(65.1 \pm 11.2$ versus $56.9 \pm 11.6 ; p=0.018)$, those treated with anxiolytics had worse sleep quality $(15.2 \pm 3.0$ versus 11.4 $\pm 3.9 ; \mathrm{p}=0.001)$ and those treated with anticonvulsants had better sleep quality ( $11.5 \pm 3.6$ versus $13.8 \pm 4.2$; $\mathrm{p}=0.039)$.

Table 1. Fibromyalgia Patients' Clinical Profile

\begin{tabular}{|c|c|}
\hline \multirow{2}{*}{ Questionnaire Scores } & FM Patients \\
\cline { 2 - 2 } & Mean (SD) \\
\hline \hline FIQ Total Score & $62.4(11.9)$ \\
\hline HAM-D Total Score & $12.2(4.4)$ \\
\hline HAM-A Total Score & $11.1(4.3)$ \\
\hline PSQI Total Score & $12.8(4.1)$ \\
\hline
\end{tabular}

FM indicates fibromyalgia; SD, standard deviation; FIQ, Fibromyalgia Impact Questionnaire; HAM-D, Hamilton Depression Rating Scale; HAM-A, Hamilton Anxiety Rating Scale; PSQI, Pittsburgh sleep quality index.

\section{Group Differences in Psychophysical Measures}

TPT, experimental pain temperature and TOL were significantly lower in FM patients, relative to HC. Pain ratings during the $\mathrm{CPT}$ were higher in $\mathrm{FM}$ patients, relative to HC. Lastly, a deficit in ICPM efficacy was observed in FM patients, relative to $\mathrm{HC}$ (Table 2). [Note: There were no differences between FM patients treated with and without antidepressants, anti-inflammatory drugs, anticonvulsants, anxiolytics and opiates on any psychophysical measure].

\section{Correlation Between Psychological Factors and Pain Measures}

In the FM group, the FIQ total score was significantly related to TOL and non-significantly to TPT and pain ratings during the CPT (Table 3). The FIQ total score remained a significant predictor of TOL when antidepressant medication was entered as a covariate using multivariate analyses. Moreover, the FIQ pain score was significantly related to mean PPT. Finally, non-significant trends also emerged for the relationships between pain ratings during the CPT and the HAM-D, HAM-A and FIQ total scores. Unfortunately, the use of hierarchical multivariate regression analyses failed to produce a model explaining a larger portion of the dependent variable's variance (e.g. pain rating during the CPT) when HAM-D, HAM-A and FIQ total scores were considered as multiple predictors $(\mathrm{p}=0.051)$.

\section{ICPM Efficacy}

We detected a significant negative relationship between ICPM efficacy and PSQI total score (Table 4; Fig. 1), and this relationship remained significant after controlling for the potential confounding influence of anticonvulsants and anxiolytics, using multivariate regression analyses. More specifically, ICPM efficacy was significantly related to two components of the PSQI: sleep efficiency ( $\mathrm{r}=-0.374$; $\mathrm{p}=0.008)$ and sedative medication use $(\mathrm{p}=-0.311 ; \mathrm{p}=0.030)$. ICPM efficacy was not significantly related to age, FIQ total score, FIQ pain score, HAM-D total score and HAM-A total score (Table 4). 
Table 2. Pain Measures in Fibromyalgia Patients and Healthy Controls

\begin{tabular}{|c|c|c|c|}
\hline Pain Measures & FM Patients Mean (SD) & HC Mean (SD) & ANOVA \\
\hline \hline Tender points $(\mathrm{Kg})(\mathrm{PPT})$ & $1.3(0.6)$ & -- & -- \\
\hline Thermal pain threshold $\left({ }^{\circ} \mathrm{C}\right)(\mathrm{TPT})$ & $39.1(3.7)$ & $43.1(3.7)$ & $\mathrm{F}=26.0 ; \mathrm{p}=0.0001$ \\
\hline Experimental pain temperature $\left({ }^{\circ} \mathrm{C}\right)$ & $44.1(2.4)$ & $46.8(1.1)$ & $\mathrm{F}=42.9 ; \mathrm{p}=0.0001$ \\
\hline Thermal pain tolerance $\left({ }^{\circ} \mathrm{C}\right)(\mathrm{TOL})$ & $44.8(3.1)$ & $47.7(1.9)$ & $\mathrm{F}=15.7 ; \mathrm{p}=0.0001$ \\
\hline Cold pressor test $(\mathrm{CPT})($ pain ratings) & $87.8(16.0)$ & $62.6(26.9)$ & $\mathrm{F}=30.2 ; \mathrm{p}=0.0001$ \\
\hline ICPM efficacy (in \%) & $0.9(49.3)$ & $29.0(26.2)$ & $\mathrm{F}=10.4 ; \mathrm{p}=0.002$ \\
\hline
\end{tabular}

CPT, cold-pressor test; FM, fibromyalgia; HC, healthy control; ANOVA, analysis of variance; PPT, pressor pain threshold; ICPM, inhibitory conditioned pain modulation.

Table 3. Correlation Analyses in Fibromyalgia Patients Between Psychophysical Measures and Clinical Variables

\begin{tabular}{|c|c|c|c|}
\hline Clinical Variables & Psychophysical Measures & r & \multicolumn{1}{|c|}{-Value } \\
\hline \hline \multirow{2}{*}{ FIQ total score } & Thermal pain tolerance (TOL) & -0.333 & 0.018 \\
\cline { 2 - 5 } & Thermal pain threshold (TPT) & -0.267 & 0.244 \\
\cline { 2 - 5 } & Cold pressor test (CPT) & -0.357 & 0.088 \\
\hline FIQ pain score & Tender points (PPT) & 0.277 & 0.011 \\
\hline HAM-D total score & Cold pressor test (CPT) & 0.274 & 0.051 \\
\hline HAM-A total score & Cold pressor test (CPT) & \multicolumn{2}{|c|}{0.054} \\
\hline
\end{tabular}

*All correlations with a level of significance of $\mathrm{p}<0.1$ are reported here; FIQ indicates Fibromyalgia Impact Questionnaire; HAM-D, Hamilton Depression Rating Scale; HAM-A, Hamilton Anxiety Rating Scale; PSQI, Pittsburgh Sleep Quality Index.

\section{DISCUSSION}

In this FM group, we observed moderate levels of anxiodepressive symptoms, poor sleep quality and low PPT, confirming that these variables are frequently associated with FM [4,6,17,41]. Moreover, our study demonstrated several differences between FM patients and $\mathrm{HC}$ in experimentally-induced pain perception. Using thermal stimuli, we found that TPT and TOL were significantly lower in FM compared to HC. Similarly, pain ratings during the CPT were higher in FM patients relative to HC. These results are consistent with several psychophysical studies that described hyperalgesia and allodynia in FM using thermal stimuli $[6,17,19]$. We also measured ICPM efficacy in both groups and found a deficit in ICPM in FM patients relative to $\mathrm{HC}$, as previously reported $[9,15,16]$.

Table 4. Correlation Analyses in FM Patients Between ICPM Efficacy and Clinical Variables

\begin{tabular}{|c|c|c|}
\hline Clinical Variables & $\mathbf{r}$ & $\boldsymbol{p}$-Value \\
\hline \hline Age & -0.094 & 0.518 \\
\hline FIQ total Score & -0.120 & 0.406 \\
\hline FIQ pain item & -0.004 & 0.980 \\
\hline HAM-D & -0.097 & 0.501 \\
\hline HAM-A & -0.050 & 0.730 \\
\hline PSQI Total Score & -0.382 & 0.006 \\
\hline
\end{tabular}

FM indicates fibromyalgia; ICPM, inhibitory conditioned pain modulation; FIQ, Fibromyalgia Impact Questionnaire; HAM-D, Hamilton Depression Rating Scale; HAM-A, Hamilton Anxiety Rating Scale; PSQI, Pittsburgh Sleep Quality Index.

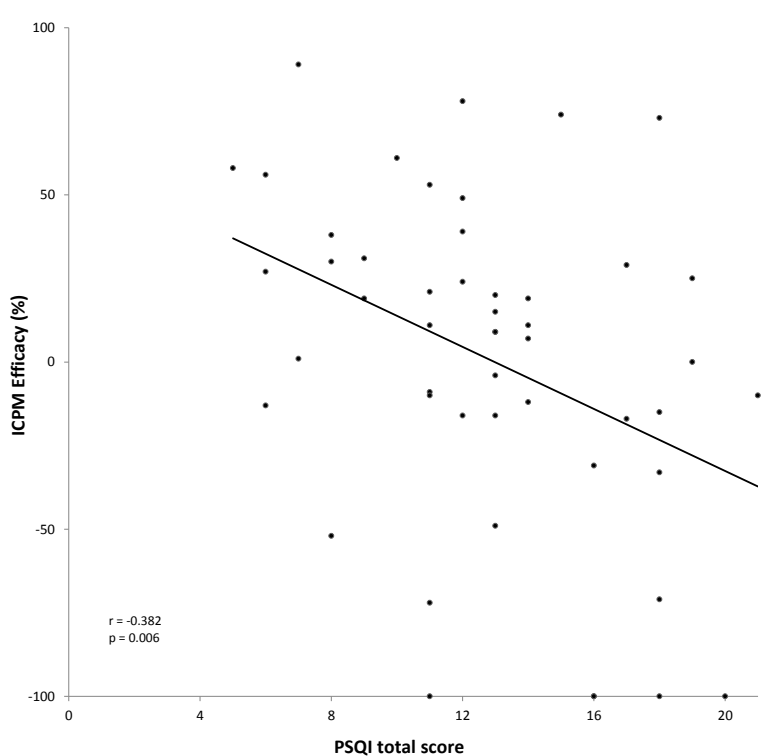

Fig. (1). The association between sleep problems and ICPM efficacy in FM. This figure illustrates a significant negative correlation between sleep problems, as measured with the PSQI (total score), and ICPM efficacy (expressed in \%) in FM. More precisely, increased sleep problems were associated with the weakest level of ICPM in this population. ICPM indicates inhibitory conditioned pain modulation; FM, fibromyalgia; PSQI, Pittsburg sleep quality index.

The main objective of this study was to establish if pain perception, especially ICPM efficacy, varies in function of 
FM symptoms, depression, anxiety, age and sleep quality in FM patients. We found that FIQ total score was significantly related to TOL. Furthermore, the FIQ pain score was correlated with PPT. Finally, pain ratings during the CPT were (non-significantly) related to HAM-D and HAM-A total scores. These results are consistent with studies showing associations between experimentally-induced pain perception and the severity of FM symptoms, as shown by other groups using mechanical $[22,42]$ and thermal noxious stimuli [6].

The most important finding of this study is the demonstration of a relationship between sleep quality and ICPM efficacy in FM. Conversely, age, depression, anxiety and FM symptoms were not significantly related to ICMP efficacy. More precisely, our study showed that the ICPM efficacy was significantly related to sleep efficiency and sedative medication use. Noteworthy, sleep problems were specifically related to ICPM efficacy, as the PSQI total score was not correlated with any other psychophysical measure. The lack of association between sleep problems and pain thresholds has been previously reported $[43,44]$. As for the relationship between ICPM efficacy and sleep impairments, it is consistent with recent studies reporting that sleep disruption can reduce ICPM efficacy. Indeed, the effect of sleep disturbance on ICPM was described in a group of healthy adults [25]. The authors studied the sleep polysomnographic activity among 32 healthy females for 7 nights. Subjects were randomized to control, forced awakening or restricted sleep opportunity conditions. A significant loss of pain inhibition was shown only in the forced awakening group. These findings suggest that sleep continuity disturbance, but not simple sleep restriction, impairs ICPM. Another study from this group showed that measures of sleep efficiency and duration are positively associated with better functioning of ICPM in patients with temporo-mandibular joint disorder [45]. As early as 1975, Modolfsky and colleagues disrupted sleep in healthy subjects and observed the next day that participants had FM-like symptoms [46,47]. In our study, it is not possible to determine the directionality of the observed correlation. It is possible that impaired ICPM engenders sleep disturbances in FM patients. Alternatively, the relationship between sleep disturbances and pain symptoms could be caused by a vicious, self-perpetuating, non-restorative sleep cycle [46]. It must be noted, finally, that ICPM efficacy was also significantly related to sedative medication use. This correlation may have emerged as significant simply because ICPM efficacy was significantly correlated with poor sleep, and that impaired sleep required treatment with sedative drugs. Consistently, we found that anxiolytic treatment was associated with poor sleep quality in our FM patients.

In contrast to a previous study from our laboratory, we did not find a relationship between depressive symptoms and ICPM efficacy in FM [17]. In that study, FM patients were separated in two groups: patients with and without depressive symptoms, based on a factor analysis [48]. In the present study, the absence of relationship can be explained by the small number of subjects suffering from MDD in the FM patients, who had low-to-moderate levels of anxiodepressive symptoms. Similarly, while previous studies demonstrated that age influences ICPM in healthy subjects [24,26], ICPM efficacy was not related to age in our study.
This finding can be explained by the small standard deviation of age in our FM patients, who were relatively old (age $=50.4 \pm 10.2$ years), whereas our previous study [24] showed that ICPM efficacy starts to decline by middle age, and continues to deteriorate thereafter.

On neurobiological grounds, our findings may be explained by common neurotransmitters involved in both sleep and ICPM, including noradrenalin (NA), serotonin (5HT) and dopamine (DA). For instance, it has been shown in animals that 5-HT and NA levels decline progressively from waking to slow-wave-sleep and then to rapid-eye-movement sleep [49]. Animal studies have also shown that sleep can be manipulated by dopaminergic mechanisms [50,51]. As for pain modulation, numerous pre-clinical studies have shown ICPM depends on the recruitment of endogenous opioids in the periaqueductal gray, which trigger the release of 5-HT from neurons localized in the raphe nuclei (medulla), which dampens nociceptive afferents at the dorsal horn of the spinal cord [52]. Noradrenergic projections from the locus coeruleus produce similar effects [53]. Finally, mesocorticolimbic dopamine pathways have also been shown to be involved in pain modulation in rodents [53] and humans [18]. It is therefore possible that DA, NA and/or 5HT alterations can impair both sleep quality and ICPM efficacy in FM. Interestingly, some studies showed that FM is characterized by abnormal 5-HT, NA and DA functioning [54-57]. Noteworthy, the periacqueductal gray matter - a key ICPM structure $[13,58]$ - has also been shown to modulate sleep states [59]. In this vein, a recent experimental study used functional magnetic resonance imaging (fMRI) to study the neural correlates of mechanically-induced pain perception in FM. Interestingly, the study showed that FM patients deactivated the rostral anterior cingulate cortex (rACC), relative to controls, and that and the brainstem, relative to controls, and that rACC activations were positively correlated with brainstem hemodynamic responses [60]. Such functional imaging results suggest that brainstem structures known to be involved in both pain inhibition and sleep regulation are dysfunctional in FM.

To our knowledge, this is the first study showing a relationship between ICPM efficacy and sleep quality in a relatively large sample of FM patients. The results from this study replicate that FM is associated with hyperalgesia and allodynia, and deficient ICPM, and that the severity of FM symptoms is correlated with thermal pain perception. Our results uniquely suggest that ICPM efficacy in FM is related to sleep quality, more precisely sleep effectiveness. If replicated, this latter finding may have several implications. First, it reinforces the importance of studying sleep problems and ICPM efficacy in FM. It is well known that FM women report poor sleep quality and fatigue [61,62]. A major concern among FM women, sleep problems may impair functional outcomes, as suggested by a recent study showing that sleep disturbances predict work disability due to musculoskeletal disorders [63]. The association between sleep problems and ICPM also suggests that impaired sleep may be closely linked to the FM pathophysiology. From a treatment perspective, as long as deficient ICPM is the consequence of poor sleep, better treatment of sleep problems may restore ICPM efficacy, and relieve the painful symptoms of FM. In the future, prospective studies will need to sort out the directionality of the relationship between sleep problems and deficient ICPM in FM, as this may 
have treatment implications. The neurobiological mechanisms underlying this association will need to be understood, using polysomnography [64], psychophysical and biomarker measures.

One potential limitation of our study is the large use of medication in FM patients, who received anxiolytics, antidepressants, anticonvulsants, anti-inflammatory drugs and opioids. However, poly-pharmacy is frequent in FM [65]. Also, we performed multivariate regression analyses to verify the influence of medications on results, and found that all associations between clinical and psychophysical measures remained significant after such statistical adjustments. Another limitation of our study is the lack of a pain questionnaire. Although the FIQ measures clinical pain, the use of a more specific clinical pain scale, such as the McGill Pain Questionnaire or the Brief Pain Inventory, would have enabled us to evaluate more precisely if clinical pain characteristics influence ICPM efficacy. However, we used the FIQ pain item and found relationships with psychophysical measures that are consistent with previous results on the topic. Also, it must be considered that the relationship between sleep and ICPM was highlighted using a subjective sleep evaluation. Future studies will need to replicate this correlation with both subjective and objective sleep quality measures. Finally, it must be noted that our finding of a relationship between sleep impairments and ICPM efficacy may depend on the paradigm that we used to measure ICPM as well as stimulus parameters [66].

\section{CONCLUSION}

The results of this study provide preliminary evidence that sleep quality is an important factor contributing to deficient ICPM in FM. Thus, it is possible that better treatment of sleep difficulties may lead to an improvement of painful symptoms in FM. Future studies will need: (i) to replicate this finding, (ii) to determine whether impaired sleep is primary or secondary to deficient ICPM, and (iii) to characterize the neurobiological mechanisms underlying this association.

\section{COFLICT OF INTERESTS}

The authors declare that they have no competing interests.

\section{ACKNOWLEDGEMENTS}

The study was funded by AstraZeneca Pharmaceuticals. S. Marchand received funding from the Canadian Institute of Health Research (CIHR), the American Fibromyalgia Syndrome Association (AFSA) and is a supported member of the Centre de Recherche Clinique Étienne-Le Bel du Centre Hospitalier Universitaire de Sherbrooke (CRC-ELBCHUS). E. Paul-Savoie is holder of a doctoral scholarship from the Canadian Institute of Health Research (CIHR). S. Potvin is a Junior 1 Young Investigator from the Quebec Funds in Health Research, and he is supported by the Centre Fernand-Seguin and the Louis-H Lafontaine Hospital Foundation. P. Bourgault is a Junior 1 Clinician Investigator from the Quebec Funds in Health Research and a supported member of the CRC-ELB-CHUS.

\section{REFERENCES}

[1] Wolfe F, Ross K, Anderson J, Russell IJ, Hebert L. The prevalence and characteristics of fibromyalgia in the general population. Arthritis Rheum 1995; 38: 19-28.

[2] Wolfe F, Snilders AMJ, Yunus MB. The American College of Rheumatology 1990 criteria for the classification of fibromyalgia: report of the Multicenter Criteria Committee. Arthritis Rheum 1990; 33:160-72.

[3] Wolfe F, Clauw DJ, Fitzcharles MA, et al. The American College of Rheumatology Preliminary Diagnostic Criteria for Fibromyalgia and Measurement of Symptom Severity. Arthritis Care Res 2010; 62: $600-10$.

[4] Burckhardt CS, Clark SR, Bennett RM. The fibromyalgia Impact Questionnaire: development and validation. J Rheumatol 1991; 18: 728-33.

[5] Giesecke T, Williams DA, Harris RE, et al. Subgrouping of fibromyalgia patients on the basis of pressure-pain thresholds and psychological factors. Arthritis Rheum 2003; 48: 2916-22.

[6] Hurtig IM, Raak RI, Kendall SA, Gerdle B, Wahren LK. Quantitative sensory testing in fibromyalgia patients and in healthy subjects: Identification of subgroups. Clin J Pain 2001; 17: 316-22.

[7] Turk DC, Okifuji A, Sinclair JD, Starz TW. Pain, disability, and physical functioning in subgroups of patients with fibromyalgia. J Rheumatol 1996; 23: 1255-62.

[8] Turk DC, Okifuji A, Sinclair JD, Starz TW. Differential responses by psychosocial subgroups of fibromyalgia syndrome patients to an interdisciplinary treatment. Arthritis Care Res 1998; 11:397-404.

[9] Julien N, Arsenault P, Marchand S. Widespread pain in fibromyalgia is associated to a deficit of endogenous pain inhibition. Pain 2005; 114: 295-302.

[10] Yarnitsky D, Arendt-Nielsen L, Bouhassira D, et al. Recommandations on terminology and practice of psychophysical DNIC testing. Eur J Pain 2010; 14: 339.

[11] Le Bars D, Dickenson AH, Besson JM. Diffuse noxious inhibitory controls (DNIC). I. Effects on dorsal horn convergent neurones in the rat. Pain 1979; 6: 283-304.

[12] Le Bars D, Dickenson AH, Besson JM. Diffuse noxious inhibitory controls (DNIC). II. Lack of effect on non-convergent neurones, supraspinal involvement and theoretical implications. Pain 1979; 6: 305-27.

[13] Basbaum AI, Fields HL. Endogenous pain control mechanisms: review and hypothesis. Ann Neurol 1978; 4: 451-62.

[14] Fields HL, Basbaum AI. Brainstem control of spinal paintransmission neurons. Annu Rev Physiol 1978; 40: 217-48.

[15] Kosek E, Hansson P. Modulatory influence on somatosensory perception from vibration and heterotopic noxious conditioning stimulation (HNCS) in fibromyalgia patients and healthy subjects. Pain 1997; 70: 41-51.

[16] Lautenbacher S, Rollman GB. Possible deficiencies of pain modulation in fibromyalgia. Clin J Pain 1997; 13: 189-96.

[17] De Souza JB, Potvin S, Goffaux P, Charest J, Marchans S. The Deficit of Pain Inhibition in Fibromyalgia Is More Pronounced in Patients With Comorbid Depressive Symptoms. Clin J Pain 2009; 25: 123-7.

[18] Potvin S, Larouche A, Normand E, de Souza JB, Grignon S, Marchand S. DRD3 Ser9Gly polymorphism is related to thermal pain perception and modulation in chronic widespread pain patients and healthy controls. J Pain 2009; 10: 969-75.

[19] Larouche A, Potvin S, Normand E, et al. No relationships between the ins del polymorphism of the serotonin transporter promoter and pain perception in fibromyalgia patients and healthy controls. Eur J Pain 2010; 14: 742-6.

[20] Petzke F, Gracely RH, Park KM, Ambrose K, Clauw DJ. What do tender points measure? Influence of distress on 4 measures of tenderness. J Rheumatol 2003; 30: 567-74.

[21] Wolfe F, Ross K, Anderson J, Russell IJ. Aspects of fibromyalgia in the general population: sex, pain threshold, and fibromyalgia symptoms. J Rheumatol 1995; 22: 151-6.

[22] Wolfe F. The relationship between tender points and fibromyalgia symptom variables: evidence that fibromyalgia is not a discrete disorder in the clinic. Ann Rheum Dis 1997; 56: 268-71.

[23] Behbehani MM. Functional characteristics of the midbrain periacqueducal gray. Prog Neurobiol 1995; 46: 575-605. 
[24] Larivière $M$, Goffaux $\mathrm{P}$, Marchand $\mathrm{S}$, Julien N. Changes in pain perception and descending inhibitory controls start at middle age in healthy adults. Clin J Pain 2007; 23: 506-10.

[25] Smith MT, Edwards RR, McCann UD, Haythornthwaite JA. The effects of sleep deprivation on pain inhibition and spontaneous pain in women. Sleep 2007; 30: 494-505.

[26] Edwards RR, Fillingim RB, Ness TJ. Age-related differences in endogenous pain modulation: a comparison of diffuse noxious inhibitory controls in healthy older and younger adults. Pain 2003; 101: $155-65$.

[27] Perrot S, Dumont D, Guillemin F, Pouchot J, Coste J. Quality of life in women with fibromyalgia syndrome: validation of the QIF, the French version of the Fibromyalgia Impact Questionnaire. J Rheumatol 2003; 30: 1054-9.

[28] Hamilton M. A rating scale for depression. J Neurol Neurosurg Psychiatry 1960; 23: 56-62.

[29] Normand E, Potvin S, Gaumond I, Cloutier G, Corbin JF, Marchand S. Pain inhibition is deficient in chronic widespread pain but normal in major depressive disorder. J Clin Psychiatry 2011; 72: 219-24.

[30] Potvin S, Sepehry AA, Stip E. Meta-analysis of depressive symptoms in dual-diagnosis schizophrenia. Aust NZ J Psychiatry 2007; 41: 792-9.

[31] Potvin S, Pampoulova T, Lipp O, Ait BL, Lalonde P, Stip E. Working memory and depressive symptoms in patients with schizophrenia and substance use disorders. Cogn Neuropsychiatry 2008; 13:357-66.

[32] Potvin S, Stip E, Lipp O, et al. Anhedonia and social adaptation predict substance abuse evolution in dual diagnosis schizophrenia. Am J Drug Alcohol Abuse 2008; 34: 75-82.

[33] Zhornitsky S, Stip E, Pampoulova T, et al. Extrapyramidal symptoms in substance abusers with and without schizophrenia and in nonabusing patients with schizophrenia. Mov Disord 2010; 25: 2188-94.

[34] Zhornitsky S, Stip E, Desfossés J, et al. Evolution of Substance use, Neurological and Psychiatric Symptoms in Schizophrenia and Substance use Disorder Patients: a 12-Week, Pilot, Case-Control Trial with Quetiapine. Front Psychiatry 2011;2: 22

[35] Hamilton $\mathrm{M}$. The assessment of anxiety states by rating. Br J Med Psychol 1959; 32: 50-5.

[36] Buysse DJ, Reynolds CF, Monk TH, Berman SR, Kupfer DJ. The Pittsburgh Sleep Quality Index: a new instrument for psychiatric practice and research. Psychiatry Res 1989; 28: 193-213.

[37] Tousignant-Laflamme Y, Pagé S, Goffaux P, Marchand S. An experimental model to measure excitatory and inhibitory pain mechanisms in humans. Brain Res 2008; 1230: 73-9.

[38] Willer JC, Debroucker T, Le Bars D. Encoding of nociceptive thermal stimuli by diffuse noxious inhibitory controls in humans. J Neurophysiol 1989; 62: 1028-38.

[39] Lopez-Munoz F, Alamo C. Monoaminergic neurotransmission: the history of the discovery of antidepressants from 1950s until today. Curr Pharm Des 2009; 15: 1563-86.

[40] Marchand S, Arsenault P. Spatial summation for pain perception: interaction of inhibitory and excitatory mechanisms. Pain 2002; 95: 201-6.

[41] Anch A, Lue F, MacLean A, Moldofsky H. Sleep physiology and psychological aspects of the fibrositis (fibromyalgia) syndrome. Can J Psychol 1991; 45: 179-84.

[42] Salli A, Yilmaz H, Ugurlu H. The relationship between tender point count and disease severity in patients with primary fibromyalgia. Rheumatol Int 2012; 32: 105-7.

[43] Agargun MY, Tekeoglu I, Gunes A, Adak B, Kara H, Ercan M. Sleep quality and pain threshold in patients with fibromyalgia. Compr Psychiatry 1999; 40: 226-8.

[44] Osorio CD, Gallinaro AL, Lorenzi-Fiho G, Lage LV. Sleep quality in patients with fibromyalgia using the Pittsburgh Sleep Quality Index. J Rheumatol 2006; 33: 1863-5.

[45] Edwards RR, Grace E, Peterson S, Klick B, Haythornthwaite JA, Smith MT. Sleep continuity and architecture: association with pain-inhibitory processes in patients with temporomandibular joint disorder. Eur J Pain 2009; 13: 1043-7.
[46] Moldofsky H, Scarisbrick P, England R, Smythe H. Musculoskeletal symptoms and Non-REM sleep disturbance in patients with fibrositis syndrome and healthy subjects. Psychosom Med 1975; 37: 341-51.

[47] Moldofsky H, Scarisbrick P. Induction of neurasthenic musculoskeletal pain syndrome by selective sleep stage deprivation. Psychosom Med 1976; 38: 35-44.

[48] De Souza JB, Goffaux P, Julien N, Potvin S, Charest J, Marchand S. Fibromyalgia subgroups: profiling distinct subgroups using the Fibromyalgia Questionnaire: a preliminary study. Rheumatol Int 2009; 29: 509-5.

[49] Shouse MN, Staba RJ, Saquib SF, Farber PR. Monoamines and sleep: microdialysis findings in pons and amygdala. Brain Res 2000; 860: 181-9.

[50] Isaac SO, Berridge CW. Wake-promoting actions of dopamine D1 and D2 receptor stimulation. J Pharmacol Exp Ther 2003; 307: 386-94.

[51] $\mathrm{Lu}$ J, Jhou TC, Saper CB. Identification of wake-active dopaminergic neurons in the ventral periaqueductal gray matter. J Neurosci 2006; 26 : 193-202.

[52] Suzuki R, Rygh LJ, Dickenson AH. Bad news from the brain: descending 5-HT pathways that control spinal pain processing. Trends Pharmacol Sci 2004; 25: 613-7.

[53] Millan MJ. Descending control of pain. Prog Neurobiol 2002; 66: 355474.

[54] Houvenagel E, Forzy G, Leloire O, Gallois P, Hary S, Hautecoeur P Cerebrospinal fluid monoamines in primary fibromyalgia. Rev Rhum Mal Osteoartic 1990; 57: 21-3.

[55] Legangneux E, Mora JJ, Spreux-Varoquaux O, Thorin I, Herrou M, Alvado G. Cerebrospinal fluid biogenic amine metabolites, plasma-rich platelet serotonin and $\left[{ }^{3} \mathrm{H}\right]$ imipramine reuptake in the primary fibromyalgia syndrome. Rheumatology 2001; 40: 290-6.

[56] Russell IJ, Vaeroy H, Javors M, Nyberg F. Cerebrospinal fluid biogenic amine metabolites in fibromyalgia/fibrositis syndrome and rheumatoid arthritis. Arthritis Rheum 1992; 35: 550-6.

[57] Wood PB, Schweinhardt P, Jaeger E, et al. Fibromyalgia patients show an abnormal dopamine response to pain. Eur J Neurosci 2007; 25: 3576-82.

[58] Willer JC, Bouhassira D, Le Bars D. Neurophysiological bases of the counterirritation phenomenon: diffuse control inhibitors induced by nociceptive stimulation. Neurophysiol Clin 1999; 29: 379-400.

[59] Sastre JP, Buda C, Kitahama K, Jouvet M. Importance of the ventrolateral region of the periaqueductal gray and adjacent tegmentum in the control of paradoxical sleep as studied by muscimol microinjections in the cat. Neuroscience 1996; 74: 415-6.

[60] Jensen KB, Kosek E, Petzke F, et al. Evidence of dysfunctional pain inhibition in Fibromyalgia reflected in rACC during provoked pain. Pain 2009; 144: 95-100.

[61] Landis CA, Frey CA, Lentz MJ, Rothermel J, Buchwald D, Shaver JL. Self-reported sleep quality and fatigue correlates with actigraphy in midlife women with fibromyalgia. Nurs Res 2003; 52: 140-7.

[62] Stuifbergen AK, Phillips L, Carter P, Morrison J, Todd A. Subjective and objective sleep difficulties in women with fibromyalgia syndrome. J Am Acad Nurse Pract 2010; 22: 548-56.

[63] Salo P, Oksanen T, Sivertsen B, et al. Sleep disturbances as a predictor of cause-specific work disability and delayed return to work. Sleep 2010; 33: 1323-31.

[64] Lavigne GJ, Okura K, Abe S, et al. Gender specificity of the slow wave sleep lost in chronic widespread musculoskeletal pain. Sleep Med 2011; 12: $179-85$

[65] Gore M, Sadosky A, Zlateva G, Clauw D. Initial use of pregabalin, patterns of pain-related pharmacotherapy, and healthcare resource use among older patients with fibromyalgia. Am J Manag Care 2010; 16: S144-53.

[66] Stevens A, Batra A, Kotter I, Bartels M, Schwarz J. Both pain and EEG response to cold pressor stimulation occurs faster in fibromyalgia patients than in control subjects. Psych Res 2000; 97: 237-47.

This is an open access article licensed under the terms of the Creative Commons Attribution Non-Commercial License (http://creativecommons.org/licenses/by-nc/ $3.0 /$ ) which permits unrestricted, non-commercial use, distribution and reproduction in any medium, provided the work is properly cited. 